${ }^{1}$ Facultad de Medicina, Hospital Clínico Universidad de Chile. ${ }^{2}$ Departamento Medicina Interna, Hospital Barros Luco, Universidad de Chile. ${ }^{3}$ Departamento de Cardiología, Hospital San Juan de Dios. ${ }^{4}$ Departamento Cardiovascular, Hospital Clínico Universidad de Sección de $M$ Nuclear, Departamento Medicina, Hospital Clínico Universidad de Chile.

Trabajo realizado como Interno de Medicina, Universidad de Chile.

Recibido el 11 de junio de 2010, aceptado el 28 de diciembre de 2010. Correspondencia: Dra. Teresa Massardo Sección Medicina Nuclear, Departamento de Medicina; Hospital Clínico Universidad de Chile.

Santos Dumont $999-1 \mathrm{E}$, Independencia, Código Postal 6531063. SantiagoChile.

Fono/fax: 56-2-7770569 E-mail: tmassardo@ redclinicauchile.cl

\section{Síndrome de Tako-Tsubo: caso clínico}

\author{
CARLOS M. RAU ${ }^{1, \mathrm{a}}$, MAX KAUFFMANN ${ }^{2}$, CARLOS L. RAU ${ }^{3}$, \\ MAURICIO CERECEDA ${ }^{4}$, GABRIEL CASTRO $^{5}$, TERESA MASSARDO 5
}

\section{Tako-Tsubo syndrome. Report of one case}

Tako-Tsubo syndrome resembles an acute myocardial infarction in symptoms, laboratory parameters and electrocardiographic changes. However, angiography does not show evidence of coronary occlusion, and typically an apical ballooning of the ventricle in systole is observed. We report a 78-year-old woman with no coronary risks factors, admitted to the emergency room due to acute chest pain and an electrocardiogram compatible with an acute coronary syndrome with ST elevation. Serum troponin and creatin-kinase (MB fraction) were elevated. An emergency coronary angiography did not show a coronary occlusion. Due to the apical ballooning observed in the left ventriculography, a probable diagnosis of Tako-Tsubo was proposed. The patient had a favorable evolution. A treadmill test, echocardiogram and myocardial perfusion SPECT, performed one month later, disclosed no abnormalities.

(Rev Med Chile 2011; 139: 348-352).

Key words: Acute coronary syndrome; Cardiac output, low; Takotsubo cardiomyopathy.
$\mathrm{E}$

n Japón, en el año 1990, Sato y $\operatorname{col}^{1}$ describieron un síndrome clínico caracterizado por dolor precordial asociado a cambios electrocardiográficos (elevación del segmento ST, prolongación del intervalo QT y depresión de la onda T) con leve aumento de marcadores de daño miocárdico y ausencia de evidencia angiográfica de oclusión de vasos coronarios.

Los autores denominaron a este síndrome "Tako-Tsubo" (STT), nombre de un recipiente de cuello angosto y base globular ancha que los pescadores japoneses empleaban para capturar pulpos, debido a su semejanza con la imagen angiográfica en sístole del ventrículo izquierdo (VI) que se presenta en la fase aguda del cuadro. El sello distintivo del STT es la diskinesia anteroapical con hiperkinesia basal del VI, trastorno contráctil que regresa a la normalidad entre una a tres semanas ${ }^{1,2}$.

Generalmente, afecta a mujeres postmenopáusicas, entre los 50 y 80 años, sin factores de riesgo cardiovascular relevantes y, con frecuencia, lo precede un estrés físico o emocional importante. La etiología es aún incierta, postulándose el espasmo arterial epicárdico, alteración microvascular, miocarditis viral, niveles elevados de catecolaminas con alteración del sistema simpático y variaciones anatómicas de la arteria descendente anterior como posibles mecanismos etiopatogénicos ${ }^{2}$.

La importancia de identificar este síndrome radica en que su presentación clínica simula un infarto agudo del miocardio (IAM), sin embargo, su pronóstico y morbimortalidad son diferentes.

\section{Caso clínico}

Mujer de 78 años, autovalente y sin factores de riesgo coronario conocidos. Consultó en Servicio de Urgencia con historia de dolor torácico izquierdo de 24 horas de evolución que apareció después de ejercicio físico moderado, sin estrés emocional aparente. Se asoció a disnea y fatigabilidad. En la evaluación clínica inicial estaba bien perfundida, con pulso de 105/min, presión arterial de 98/57 
mmHg. El examen cardiovascular fue normal. En los exámenes de rutina destacaron un electrocardiograma que mostró supradesnivel del segmento ST en pared anterior (V2-V4) (Figura 1a) y marcadores de daño miocárdico elevados (troponinas 7,10 ng/ml, CK-total 368 U/L y CK-MB 57 U/L).

Se realizó coronariografía de urgencia una hora posterior al ingreso, que reveló arteria descendente anterior con lesión proximal de 30\% y flujo lento, no presentando el resto de las arterias coronarias lesiones significativas (Figura 2a-b). En la ventriculografía izquierda con contraste se observó balonamiento apical (Figura 2c-d). Se planteó como probable diagnóstico STT e ingresó a Unidad Coronaria para tratamiento médico estándar de síndrome coronario agudo (SCA).

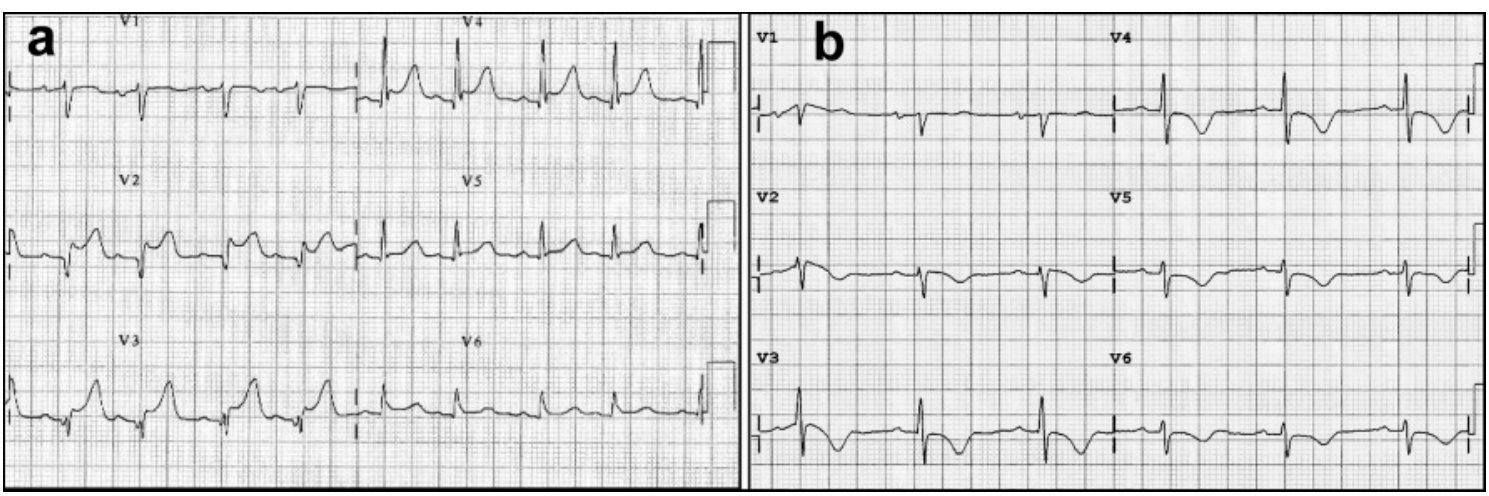

Figura 1. Electrocardiograma (ECG) de ingreso, a 24 horas del inicio del dolor torácico, muestra SDST en V2 a V4 (a). ECG al tercer día de hospitalización muestra inversión de onda T de V2 a V6 (b).

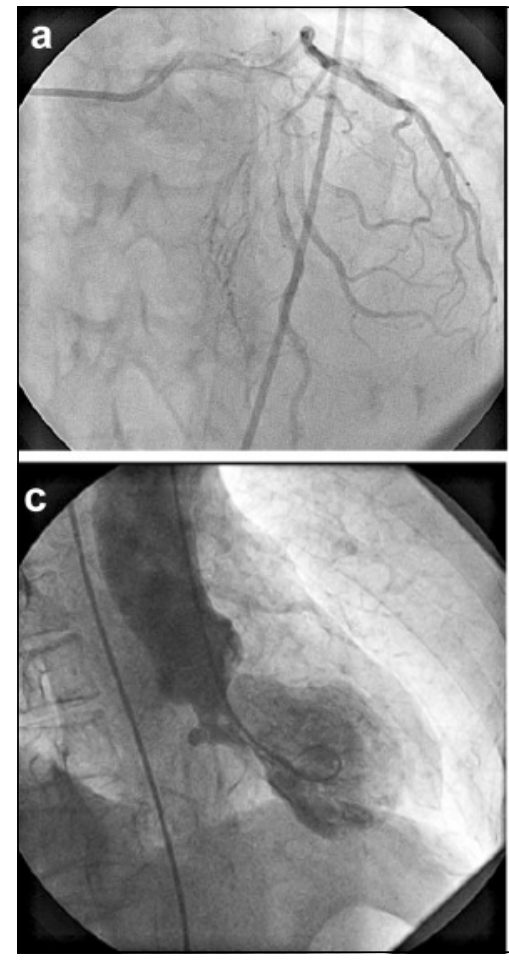

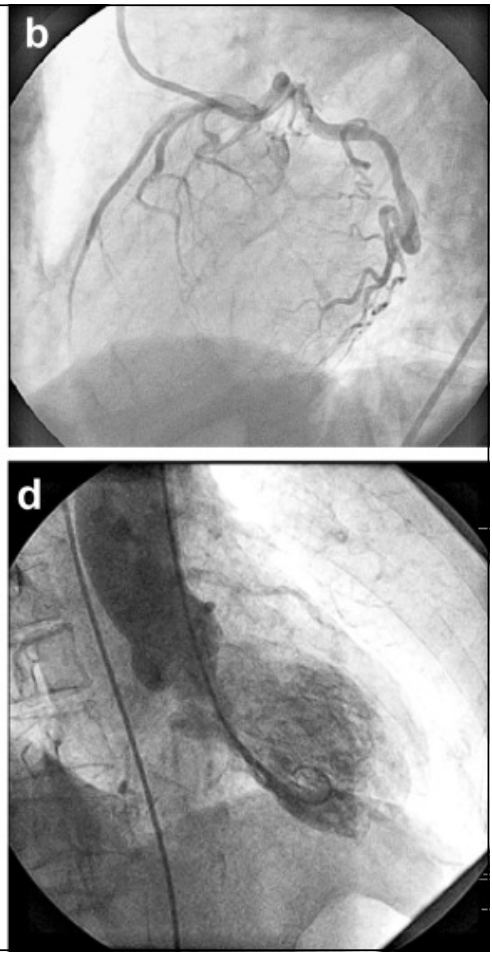

Figura 2. Imágenes invasivas iniciales: Coronariografía sin evidencias de oclusión coronaria (a y b). Ventriculografía izquierda en proyección oblicua anterior derecha en sístole (c) y en diástole (d) se observa la clásica imagen de balonamiento apical. 


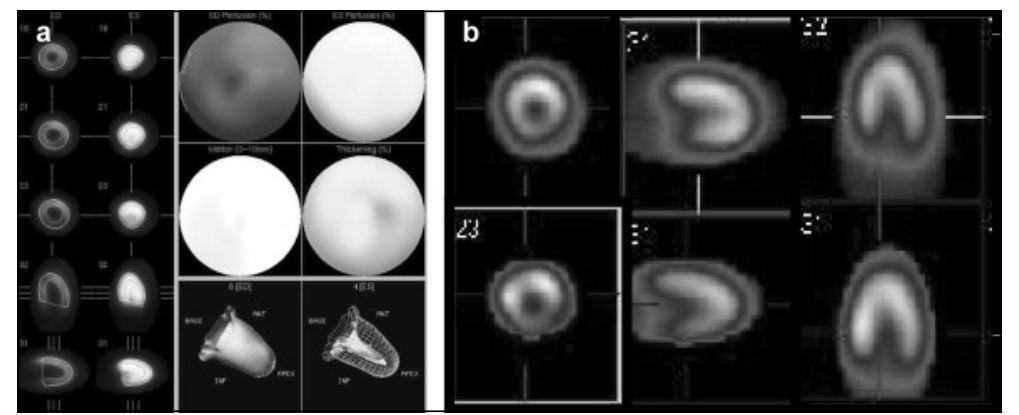

Figura 3. SPECT de perfusión miocárdica en estrés y reposo, dentro de límites normales realizado como control al mes del evento. Imagen gatillada en estrés muestra excelente contracción de todas las paredes del VI (a). Cortes tomográficos seleccionados en eje corto y largos vertical y horizontal en estrés (arriba) y reposo (abajo) no muestran alteraciones de perfusión ni cambios entre las fases (b).
La ecocardiografía en el segundo día de hospitalización mostró: 1) VI de dimensiones basales en rangos normales con extensa akinesia anteroseptoapical y de la punta; 2) Función sistólica global moderadamente deprimida con una fracción de eyección del VI estimada en $40 \%$; 3 ) Insuficiencia tricuspídea severa e insuficiencia mitral moderada; 4) Hipertensión pulmonar severa con presión sistólica de arteria pulmonar de $72 \mathrm{mmHg}$.

Al tercer día, el control electrocardiográfico reveló inversión de onda $\mathrm{T}$ de derivaciones $\mathrm{V} 2$ a V6 (Figura 1b).

La evolución posterior fue favorable, con desaparición del dolor y disminución de los marcadores de daño miocárdico por lo que se decidió el alta al noveno día hospitalización. El seguimiento al mes con ecocardiografía 2-D mostró: 1) Función sistólica de VI conservada; 2) Insuficiencia mitral leve con válvula de aspecto normal. Se efectuó estudio de perfusión miocárdica SPECT gatillado para evaluar función ventricular con Tecnecio ${ }^{99 \mathrm{~m}_{-}}$ SESTAMIBI en estrés con protocolo de Bruce y en reposo; concluyó motilidad y engrosamiento global y segmentario del VI conservados, con excelente función sistólica (Figura 3).

\section{Discusión}

La etiopatogenia del STT permanece incierta y se han postulado diversas hipótesis, entre las cuales se considera que las catecolaminas tienen un papel importante; algunos estudios plantean que corresponde a atontamiento miocárdico mediado por ellas, secundario a estrés emocional o psicológico ${ }^{3}$. Se ha demostrado que los niveles de catecolaminas están marcadamente elevados en pacientes con STT respecto a infarto de mio- cardio Killip III ${ }^{4}$. También se ha sugerido como mecanismo al espasmo coronario, sin embargo, su inducción con acetilcolina o ergonovina no es concluyente ${ }^{2}$. Otra hipótesis se refiere a alteraciones en la microcirculación coronaria aunque sin causalidad directa demostrada ${ }^{5}$. La miocarditis puede presentarse con características similares a las del STT, aunque resultados negativos en biopsias miocárdicas y en anticuerpos antivirales, la hace poco probable como etiología ${ }^{6}$.

En relación a la alteración de motilidad característica del STT, diversas explicaciones se han propuesto en relación al compromiso apical del VI. Este es estructuralmente vulnerable, pues no presenta las tres capas del miocardio, tiene menor reserva elástica, puede fácilmente presentar isquemia debido a su limitada circulación y es más sensible al estímulo adrenérgico, factores que podrían explicar la sensibilidad a la descarga catecolaminérgica ${ }^{7}$.

La explicación de la mayor incidencia en mujeres tampoco es clara; podría estar relacionada con disfunción endotelial postmenopáusica asociada a bajo nivel estrogénico ${ }^{8} \mathrm{y}$ a reactividad vasomotora en la microcirculación en respuesta al estímulo mediado por catecolaminas?

Es importante destacar que 7-10\% de los pacientes con diagnóstico inicial de SCA no presentan lesiones coronarias significativas y en muchas ocasiones es difícil establecer un diagnóstico preciso $^{10,11}$. También es esencial el diagnóstico diferencial con IAM y miocarditis, por su implicancia pronóstica y terapéutica. La resonancia magnética cardiaca permitiría identificar la etiología en un alto porcentaje de pacientes con diagnóstico inicial de SCA y coronarias angiográficamente normales; el STT se caracteriza por ausencia de realce tardío tras administración de gadolinio ${ }^{12,13}$. Asimismo, 
otros estudios no invasivos tienen un papel en la confirmación de la alteración inicial y en su resolución. Se ha demostrado mayor tamaño del defecto segmentario inicial al estudiar metabolismo con ácidos grasos respecto a trazadores de perfusión radiomarcados ${ }^{14}$.

En cuanto al manejo del STT, se plantea que inicialmente y hasta la realización de una coronariografía con ventriculografía de contraste -prueba diagnóstica de elección- se debiera iniciar tratamiento como en IAM, especialmente en población caucásica; se han descrito ciertas diferencias electrocardiográficas raciales entre STT e IAM ${ }^{15}$. Diagnosticado el cuadro, se debe efectuar soporte hemodinámico convencional para la insuficiencia cardiaca congestiva, si la hubiere, y considerar anticoagulación para prevenir formación de trombos murales, especialmente en presencia de disfunción sistólica severa; además, monitorizar arritmias auriculares y ventriculares, insuficiencia cardiaca y complicaciones mecánicas ${ }^{16,17}$.

Los resultados a corto plazo del STT son excelentes, con resolución completa ${ }^{18}$; sin embargo, no hay datos claros del pronóstico a largo plazo. Respecto a una eventual persistencia de las alteraciones segmentarias, en relación a disturbios de catecolaminas, existe evidencia de alteración apical precoz y hasta 6 meses mediante imágenes con metayodobenzilguanidina marcada ${ }^{19}$. En pacientes con STT sometidos a estrés mental, al menos 1 mes post inicio del cuadro, se demostró dilatación post-estrés y alteraciones de motilidad segmentaria transitoria en la mayoría de los casos con STT respecto a grupo control, sin alteraciones coronarias $^{20}$.

Finalmente, podemos concluir que este caso clínico muestra en forma clara la evolución favorable de una paciente con STT, cuadro aún no muy reconocido en nuestro medio, que tuvo resolución espontánea completa, en concordancia con lo descrito en la literatura.

\section{Referencias}

1. Sato H, Tateishi H, Uchida T, Dote K, Ishihara M. Takotsubo-like left ventricular dysfunction due to multivessel coronary spasm. In: Kodama K, Haze K, Hori M, Eds., Clinical Aspect of Myocardial Injury: From Ischemia to Heart Failure. Tokyo, Japan. Kagakuhyoronsha Publishing Co.; 1990. p. 56-64. (in Japanese).

2. Dote K, Sato H, Tateishi H, Uchida T, Ishihara M.
Myocardial stunning due to simultaneous multivessel coronary spasms: a review of 5 cases. J Cardiol 1991; 21: 203-14.

3. Ibáñez B, Navarro F, Farré J, Marcos-Alberca P, Orejas M, Rábago R, et al. Tako-Tsubo transient left ventricular apical ballooning is associated with a left anterior descending coronary artery with a long course along the apical diaphragmatic surface of the left ventricle. Rev Esp Cardiol 2004; 57: 209-16.

4. Wittstein IS, Thiemann DR, Lima JAC, Baughman KL, Schulman SP, Gerstenblith G, et al. Neurohumoral features of myocardial stunning due to sudden emotional stress. N Engl J Med 2005;352: 539 -48.

5. Ako J, Takenaka K, Uno K, Nakamura F, Shoji T, Iijima $\mathrm{K}$, et al. Reversible left ventricular systolic dysfunction reversibility of coronary microvascular abnormality. Jpn Heart J 2001; 42: 355-63.

6. Kurisu S, Sato H, Kawagoe T, Ishihara M, Shimatani Y, Nishioka K, et al. Tako-Tsubo-like left ventricular dysfunction with ST segment elevation: a novel cardiac syndrome mimicking acute myocardial infarction. Am Heart J 2002; 143: 448-55.

7. Mori H, Ishikawa S, Kojima S, Hayashi J, Watanabe Y, Hoffman JI, Okino H. Increased responsiveness of left ventricular apical myocardium to adrenergic stimuli. Cardiovasc Res 1993; 27: 192-8.

8. Ueyama T, Hano T, Kasamatsu K, Yamamoto K, Tsuruo Y, Nishio I. Estrogen attenuates the emotional stressinduced cardiac responses in the animal model of Tako-tsubo (Ampulla) cardiomyopathy. J Cardiovasc Pharmacol 2003; 42 Suppl 1:S117-9.

9. Celermajer DS, Sorensen KE, Spiegelhalter DJ, Georgakopoulos D, Robinson J, Deanfield JE. Aging is associated with endothelial dysfunction in healthy men years before the age-related decline in women. J Am Coll Cardiol 1994; 24: 471-6.

10. Wang K, Asinger RW, Marriot HJL. ST-segment elevation in conditions other than acute myocardial infarction. N Engl J Med 2003; 348:2128-35

11. Larson DM, Menssen KM, Sharkey SW, Duval S, Schwartz RS, Harris J, et al. "False-positive" cardiac catheterisation laboratory activation among patients with suspected ST segment elevation myocardial infarction. JAMA 2007;298: 2754-60.

12. Teraoka K, Kiuchi S, Takada N, Hirano M, Yamashina A. Images in cardiovascular medicine. No delayed enhancement on contrast magnetic resonance imaging with Takotsubo cardiomyopathy. Circulation 2005; 111:e2612.

13. Mitchell JH, Hadden TB, Wilson JM, Achari A, Muthupillai R, Flamm SD. Clinical features and usefulness 
of cardiac magnetic resonance imaging in assessing myocardial viability and prognosis in Takotsubo cardiomyopathy (transient left ventricular apical ballooning syndrome). Am J Cardiol 2007;100: 296-301.

14. Kurisu S, Inoue I, Kawagoe T, Ishihara M, Shimatani Y, Nishioka K, et al. Myocardial Perfusion and Fatty Acid Metabolism in Patients With Tako-Tsubo-Like Left Ventricular Dysfunction. J Am Coll Cardiol 2003; 41: 743-8.

15. Núñez-Gil IJ, Luaces M, García-Rubira JC, Zamorano J. Electrocardiographic Criteria in Takotsubo Cardiomyopathy and Race Differences. Asians Versus Caucasians. J Am Coll Cardiol 2010;56:1433-4

16. Bybee KA, Kara T, Prasad A, Lerman A, Barsness GW, Wright RS, Rihal CS. Systematic review: transient left ventricular apical ballooning: a syndrome that mimics ST-segment elevation myocardial infarction. Ann Intern Med 2004; 141: 858-65.

17. Tsuchihashi K, Ueshima K, Uchida T, Oh-mura N, Kimura K, Owa M, et al. Transient left ventricular apical ballooning without coronary artery stenosis: a novel heart syndrome mimicking acute myocardial infarction. Angina Pectoris-Myocardial Infarction Investigations in Japan. J Am Coll Cardiol 2001; 38: 11-8.

18. Seth PS, Aurigemma GP, Krasnow JM, Tigue DA, Untereker WJ, Meyer TE. A syndrome of transient left ventricular apical wall motion abnormality in the absence of coronary disease: a perspective from the United States. Cardiology 2003; 100: 61-6.

19. Moriya M, Mori H, Suzuki N, Hazama M, Yano K. Sixmonth follow-up of takotsubo cardiomyopathy with I-123-beta-metyl-iodophenyl pentadecanoic acid and I-123-meta-iodobenzyl-guanidine myocardial scintigraphy. Intern Med 2002; 41: 829-33.

20. Sciagrà R, Parodi G, Del Pace $S$, Genovese $S$, Zampini L, Bellandi B, et al. Abnormal response to mental stress in patients with Takotsubo cardiomyopathy detected by gated single photon emission computed tomography. Eur J Nucl Med Mol Imaging 2010; 37: 765-72. 